\title{
MODELOS DE PREDICCIÓN DE RENDIMIENTO DE CANOLA EN FUNCIÓN DEL CONTENIDO DE HUMEDAD DISPONIBLE EN EL SUELO
}

\author{
MODELLING CANOLA YIELD UNDER DIFFERENT SOIL WATER CONTENTS
}

\author{
Marco A. Inzunza-Ibarra ${ }^{1 \star}$, Ernesto A. Catalán-Valencia ${ }^{1}$, M. Magdalena Villa-Castorena ${ }^{1}$, \\ Ignacio Sánchez-Cohen ${ }^{1}$, Ernesto Sifuentes-Ibarra ${ }^{2}$ y Abel Román-López ${ }^{1}$
}

${ }^{1}$ Centro Nacional de Investigación Disciplinaria en Relación Agua Suelo Planta Atmósfera (CENID RASPA), Instituto Nacional de Investigaciones Forestales, Agrícolas y Pecuarias (INIFAP). Km 6.5 Canal Sacramento, Margen derecha. 35140, Gómez Palacio, Dgo., México. Tel. y Fax 01(871)159-0104. Fax ext.106. ${ }^{2}$ C. E. Valle del Fuerte, INIFAP. Km 1609 Carr. internacional México-Nogales, Juan José Ríos. 81110, Guasave, Sinaloa.

${ }^{*}$ Autor para correspondencia (inzunza.marco@inifap.gob.mx)

\section{RESUMEN}

La canola (Brassica napus L.) es un cultivo de gran demanda por su aceite de alta calidad para consumo humano y por ser alternativa viable en la rotación de cultivos por sus reducidas necesidades hídricas. El estudio se realizó en Gómez Palacio, Durango en 2009, con el objetivo de determinar un modelo matemático que prediga confiablemente a la producción de grano de la canola ante diferentes condiciones de estrés hídrico en el suelo en dos etapas de su crecimiento. Se ensayaron siete tratamientos resultantes del diseño de tratamientos San Cristóbal para dos factores: humedad aprovechable consumida del suelo (HAC) [40-40, 40-80, 60-60, 60-100, 80-40, 80-80, y 100-60], en combinación con dos etapas fenológicas (vegetativa, que comprende de emergencia a inicio de floración; y reproductiva, de inicio de floración a madurez fisiológica). Los tratamientos se distribuyeron en forma aleatoria en un diseño experimental de bloques al azar con cuatro repeticiones. Las variables medidas fueron: rendimiento de grano $\left(\mathrm{kg} \mathrm{ha}^{-1}\right)$, eficiencia de uso de agua de la canola $\left(\mathrm{kg} \mathrm{m}^{-3}\right)$, evapotranspiración del cultivo, y régimen efectivo de humedad en el suelo. Los resultados mostraron que la canola alcanza los mayores valores de rendimiento de grano y de eficiencia de uso del agua $\left(3.3 \mathrm{t} \mathrm{ha}^{-1} \mathrm{y}\right.$ de $\left.0.585 \mathrm{~kg} \mathrm{~m}^{-3}\right)$, al desarrollarse con 65 y $61 \%$ de HAC en las etapas vegetativa y reproductiva, respectivamente. Los modelos de predicción mostraron que la canola maximiza su producción de grano $\left(3.15 \mathrm{t} \mathrm{ha}^{-1}\right)$ al consumir láminas de agua de 21 y $35 \mathrm{~cm}$, en las dos etapas fenológicas citadas.

Palabras clave: Brassica napus, evapotranspiración, humedad aprovechable residual, régimen de humedad.

\section{SUMMARY}

Canola oil has an elevated demand in the market because of its high quality for human consumption, and because the plant (Brassica napus L.) it is a viable alternative for crop rotation due to its low water requirements. This study was done at Gómez Palacio, Durango, in 2009, with the aim to find math reliable models to predict grain production under different soil water stress regimes referred to water consumption at two developmental stages. Following the San Cristobal Treatment Design, seven treatments of available soil moisture content (HAC, \%): [40-40, 40-80, 60-60, 60-100, 80-40, 80-80, and 100-60] in combination with two development stages (vegetative, from emergence to beginning of flowering; and reproductive from the beginning of flowering to physiological maturity), were tested. The experimental design was a randomized block with four replicates. Measured variables were: grain yield $\left(\mathrm{kg} \mathrm{ha}^{-1}\right)$, water use efficiency $\left(\mathrm{kg} \mathrm{m}^{-3}\right)$, evapotranspiration $(\mathrm{mm})$, and soil moisture depletion (\%). Results showed that the highest grain yield and water use efficiency $\left(3.3 \mathrm{tha}^{-1}\right.$ and $\left.0.585 \mathrm{~kg} \mathrm{~m}^{-3}\right)$ were obtained when canola was grown under 65 and $61 \% \mathrm{HAC}$ at vegetative and reproductive stages, respectively. Prediction models showed that canola maximizes grain yield $\left(3.15 \mathrm{t} \mathrm{ha}^{-1}\right)$ when consumed 21 and $35 \mathrm{~cm}$ of water during the vegetative and reproductive developmental stages, respectively.

Index words: Brassica napus, evapotranspiration, availabe soil moisture, soil water regime.

\section{INTRODUCCIÓN}

México tiene en forma aproximada $50 \%$ de su superficie árida o semiárida, con una precipitación anual de 100 a 500 $\mathrm{mm}$, en donde la aplicación de riego se hace necesaria para una producción agrícola rentable (CONAGUA, 2011). La investigación sobre el riego en estas áreas ha tenido como principal enfoque la optimización de cuánto y cuándo regar las áreas de riego, con el objetivo de maximizar la producción por unidad de volumen de agua aplicado. Esto significa incrementar la relación entre la producción de semilla o materia seca y la cantidad de agua evapotranspirada (Blum, 2005).

El cultivo de la canola (Brassica napus L.) presenta características genéticas que le permiten incrementar tal relación mediante la tecnología adecuada de riego (Si y Walton, 2004). Además, la respuesta del rendimiento de grano de canola al estrés hídrico depende del estado de desarrollo $\mathrm{y}$ de las condiciones de humedad que ocurran antes y durante a la floración (Gan et al., 2004). Según Johnston et al. (2002), por arriba de un consumo de agua mínimo de 12.7 $\mathrm{cm}$ (lámina de riego) la producción de grano de canola se incrementa a una tasa de 69 a $72 \mathrm{~kg} \mathrm{ha}^{-1} \mathrm{~cm}^{-1}$, y el estrés de agua durante la floración influye en forma adversa a la formación de vainas y en el tamaño de la semilla, lo que al final del ciclo resulta en baja producción de semilla.

En Irán, Faraji et al. (2009) estudiaron por dos años el 
efecto de altas temperaturas en combinación con fechas de siembra y riegos complementarios, en el rendimiento de semilla de canola y en su eficiencia de uso del agua en un clima mediterráneo semiárido en Irán. Sus resultados mostraron que el riego complementario fue eficiente para reducir el estrés por déficit de agua y para incrementar el rendimiento de grano y la producción de materia seca del cultivo. Además reportaron una fuerte correlación negativa entre el rendimiento de semilla y la temperatura del aire durante la etapa reproductiva. Por ello la fecha óptima de siembra se asoció con altos índices de área foliar y rendimiento de materia seca y bajas temperaturas durante el periodo reproductivo.

Por su parte, Tesfamariam et al. (2010) reportaron que el estrés de agua durante la floración de la canola retrasó su maduración por un periodo equivalente a 114 grados día de desarrollo (GDD), que se calculó con el método de Monteith (1977) con temperatura base de $5{ }^{\circ} \mathrm{C}$, mientras que el estrés hídrico durante llenado de grano adelantó la maduración hasta en 127 GDD. El tratamiento sin restricción de humedad alcanzó el más alto índice de área foliar con un valor de 8, un uso del agua de $709 \mathrm{~mm}$, un rendimiento de semilla de $3831 \mathrm{~kg} \mathrm{ha}^{-1}$, y un contenido de aceite en la semilla de $398 \mathrm{~g} \mathrm{~kg}^{-1}$. Al ser sometida a déficit hídrico durante la floración, la canola mostró el más bajo rendimiento de semilla con $1361 \mathrm{~kg} \mathrm{ha}^{-1}$, un contenido de aceite en la semilla de $340 \mathrm{~g} \mathrm{~kg}^{-1}$, y un consumo de agua de $332 \mathrm{~mm}$. Por lo anterior, Tesfamariam et al. (2010) concluyeron que la producción de semilla y aceite de canola fueron más sensibles al estrés hídrico en la etapa de floración que durante las etapas vegetativa y de llenado de grano.

La canola se caracteriza por tener bajas tasas de evapotranspiración como consecuencia a su alta capacidad de autorregulación bajo condiciones restrictivas de humedad, mediante el cierre parcial o total de los estomas (Al-Ghobari, 2000; Chongo y Mc Vetty, 2000), característica que la hacen altamente competitiva para su explotación en áreas con un restringido recurso de agua para riego. Al respecto, Kassam et al. (2007) afirmaron que es fundamental conocer la relación entre el rendimiento de este cultivo y la cantidad de agua utilizada, así como su eficiencia de uso del agua, para optimizar el uso del agua y maximizar la producción de grano.

En la Comarca Lagunera, mediante una función de predicción para la canola cultivada con riego para su ciclo completo, Inzunza et al. (2010) concluyeron que el mayor rendimiento de grano de $3.1 \mathrm{t} \mathrm{ha}^{-1}$ se logra cuando el cultivo se desarrolla bajo una condición hídrica de $65 \%$ de la humedad aprovechable en el suelo y en todo su ciclo consume $48 \mathrm{~cm}$ de agua por evapotranspiración.
En complemento a este estudio previo, la presente investigación se diseñó para evaluar variantes del régimen hídrico en dos etapas fenológicas del cultivo, y su viabilidad para incorporarlo a la rotación de cultivos de la Región Lagunera y a otras regiones agrícolas de características climáticas similares, ya que además de su aceite de alta calidad para consumo humano, la canola es una excelente opción para disminuir plagas y enfermedades del suelo (Booth y Gunstone, 2004).

Por lo anterior, este estudio se enfocó en determinar la relación óptima entre la producción de grano del cultivo y la variación del régimen de humedad en las etapas vegetativa y reproductiva, y encontrar un modelo que relacione el rendimiento de grano de la canola con las diferentes tasas de evapotranspiración que se generaran en cada régimen de humedad.

\section{MATERIALES Y MÉTODOS}

\section{Condiciones experimentales de clima y suelo}

El estudio se hizo durante el ciclo agrícola invierno-primavera 2008-2009, en terrenos del Ejido Las Huertas del municipio de Gómez Palacio, Durango, en la Comarca Lagunera. El sitio experimental se localiza en las coordenadas geográficas $25^{\circ} 31^{\prime} 57^{\prime \prime} \mathrm{LN}$ y $103^{\circ} 25^{\prime} 57^{\prime \prime} \mathrm{LO}$, a una altitud de $1139 \mathrm{~m}$.

El clima de la Comarca Lagunera es de tipo desértico con bajo contenido de humedad atmosférica, precipitación pluvial promedio entre 200 y $300 \mathrm{~mm}$ anuales en la zona agrícola de riego y de 400 a $500 \mathrm{~mm}$ en la zona montañosa Oeste, con evaporación media anual de $2600 \mathrm{~mm}$ y temperatura media anual de $20^{\circ} \mathrm{C}$. Tanto la llanura agrícola como gran parte de la zona montañosa presentan dos periodos térmicos bien definidos: el primero comprende siete meses, de abril a octubre, en que la temperatura media mensual excede de $20^{\circ} \mathrm{C}$; el segundo abarca de noviembre a marzo, en el que la temperatura media mensual varía de 13.6 a 19.5 ${ }^{\circ} \mathrm{C}$. Los meses más fríos son diciembre y enero, y en este último mes se registra el promedio más bajo de temperatura, de $5.8^{\circ} \mathrm{C}$ aproximadamente (Villa-Castorena et al., 2005a). Según García (1988), su clima se clasifica como BW(h’)h $\mathrm{w}(\mathrm{e})$, que corresponde a muy árido, cálido con temperatura media anual de $22^{\circ} \mathrm{C}$ y temperatura media del mes más frío de $18^{\circ} \mathrm{C}$; su periodo libre de heladas es abril a octubre, y pueden ocurrir granizadas en mayo; las lluvias son en verano, con un porcentaje de lluvias invernales que va de 5 a $10.2 \%$.

Para caracterizar el suelo del lote experimental, se hizo un muestreo a tres profundidades $(0-30,30-60$ y $60-90 \mathrm{~cm})$, con cuatro repeticiones. Las características químicas determinadas fueron: disponibilidad de nitratos (método de 
Kjeldahl), de fósforo (método de Duval) y potasio (espectrofotometría), carbonatos totales (volumetría), conductividad eléctrica (conductimetría), capacidad de intercambio catiónico (con extracción de yeso), y pH (pasta de saturación), cuyos valores se muestran en el Cuadro 1.

Las características físicas del suelo evaluadas fueron: contenido de materia orgánica (método de Walkley y Black); proporción de arena, limo y arcilla en la textura (método de la pipeta de Robinson); capacidad de campo y punto de marchitez permanente (métodos de la olla y membrana de presión, respectivamente); y densidad aparente (método del cilindro) (Plenecassage et al., 1977). Sus valores se muestran en el Cuadro 2.

Según la clasificación de Castellanos et al. (2000), este suelo presentó baja disponibilidad de nitratos, moderadamente baja de fósforo y alta de potasio, bajos contenidos de sales solubles, de sodio y de materia orgánica. Por su $\mathrm{pH}$ promedio de 8.5 se considera un suelo alcalino que podría causar problemas de asimilación de Fe, Mn y Zn, pero al ser irrigado es un suelo de alta productividad y sin deficiencias importantes de estos micro-nutrimentos.

\section{Tratamientos de régimen hídrico}

Se probaron siete combinaciones de abatimiento de la humedad aprovechable consumida en el suelo (HAC), resultantes de la matriz de tratamientos San Cristóbal para dos factores 4 x 4 (Martínez, 1988; Villasmil et al., 1974). Tales variantes de HAC se aplicaron en dos etapas, de emer- gencia a inicio de floración (vegetativa) y de inicio de floración a madurez fisiológica (reproductiva). Los niveles de cada factor fueron 40, 60, 80 y $100 \%$ de la HAC en cada etapa fenológica (Cuadro 3). El riego se aplicó cuando en el perfil de $0-90 \mathrm{~cm}$ del suelo la humedad aprovechable se abatió a los niveles establecidos en los tratamientos estudiados. El abatimiento de la humedad del suelo en cada tratamiento se determinó con el método gravimétrico mediante barrenas tipo vehimeyer. Los tratamientos se distribuyeron en forma aleatoria en un diseño experimental de bloques al azar con cuatro repeticiones, con unidades experimentales de $4 \mathrm{~m}$ de ancho y $5 \mathrm{~m}$ de largo, para un área de $20 \mathrm{~m}^{2}$ por parcela.

\section{Manejo experimental}

La siembra de la canola se hizo el 23 de diciembre con el híbrido 'Hyola-401', a una densidad de siembra de $2 \mathrm{~kg}$ de semilla ha ${ }^{-1}$ distribuida en surcos de $80 \mathrm{~cm}$ de ancho y una separación entre plantas de aproximadamente $5 \mathrm{~cm}$, para obtener una población de 250 mil plantas por ha. Con base en el muestreo inicial de suelos, se fertilizó con la fórmula $120 \mathrm{~N}-60 \mathrm{P}-00 \mathrm{~K}$, la mitad aplicada al momento de la siembra en húmedo y el resto en el primer riego de auxilio(Figura 1).

Para la aplicación del riego se instaló tubería presurizada desde la fuente de abastecimiento hasta el área experimental. Para regar las melgas circunvecinas al área experimental, la tubería de conducción fue de $15.24 \mathrm{~cm}$ de diámetro con hidrantes cada $36 \mathrm{~m}$, a la cual se conectó tubería de $38.1 \mathrm{~mm}$ de diámetro con conexiones para la unión de una

Cuadro 1. Características químicas del suelo del sitio experimental.

\begin{tabular}{lccccccc}
\hline Prof. $(\mathrm{cm})$ & $\mathrm{NO}_{3}\left(\mathrm{mg} \mathrm{kg}^{-1}\right)$ & $\mathrm{P}\left(\mathrm{mg} \mathrm{kg}^{-1}\right)$ & $\mathrm{K}(\mathrm{mg} \mathrm{kg})^{-1}$ & $\mathrm{CO}_{3}\left(\mathrm{~g} \mathrm{~kg}^{-1}\right)$ & $\mathrm{CEe}\left(\mathrm{dS} \mathrm{m}^{-1}\right)$ & $\begin{array}{c}\mathrm{CIC} \\
\left(\mathrm{cmol} \mathrm{kg}^{-1}\right)\end{array}$ & $\mathrm{pH}$ \\
\hline $0-30$ & 23.18 & 9.20 & 1045.83 & 53.0 & 0.79 & 24.4 & 8.47 \\
$30-60$ & 37.27 & 8.23 & 912.50 & 68.0 & 1.07 & 23.5 & 8.30 \\
$60-90$ & 43.42 & 9.67 & 833.30 & 86.6 & 1.03 & 23.4 & 8.15 \\
\hline
\end{tabular}

$\mathrm{NO}_{3}$ = nitrato disponible; $\mathrm{P}$ = fósforo disponible; $\mathrm{K}$ = potasio disponible; $\mathrm{CO}_{3}=$ carbonatos totales; $\mathrm{CEe}$ = conductividad eléctrica; CIC = capacidad de intercambio catiónico.

Cuadro 2. Características físicas del suelo del sitio experimental.

\begin{tabular}{ccccccccc}
\hline Prof. $(\mathrm{cm})$ & MO (\%) & Arena (\%) & Limo (\%) & Arcilla (\%) & Textura & CC (\%) & PMP (\%) & Da $\left(\mathrm{g} \mathrm{cm}^{-3}\right)$ \\
\hline $0-30$ & 1.21 & 20.0 & 38.0 & 24.0 & $\begin{array}{c}\text { Franco } \\
\text { arcillo } \\
\text { limoso }\end{array}$ & 27.9 & 15.9 & 1.3 \\
$30-60$ & 0.88 & 19.6 & 47.4 & 33.0 & $\begin{array}{c}\text { Franco } \\
\text { arcillo } \\
\text { Limoso }\end{array}$ & 27.7 & 15.5 & 1.2 \\
$60-90$ & 0.82 & 17.0 & 50.0 & 33.0 & Franco & 27.9 & 15.2 & 1.3 \\
\hline
\end{tabular}

$\mathrm{MO}=$ materia orgánica; $\mathrm{CC}=$ capacidad de campo; $\mathrm{PMP}=$ punto de marchitez permanente; $\mathrm{Da}=$ densidad aparente. 


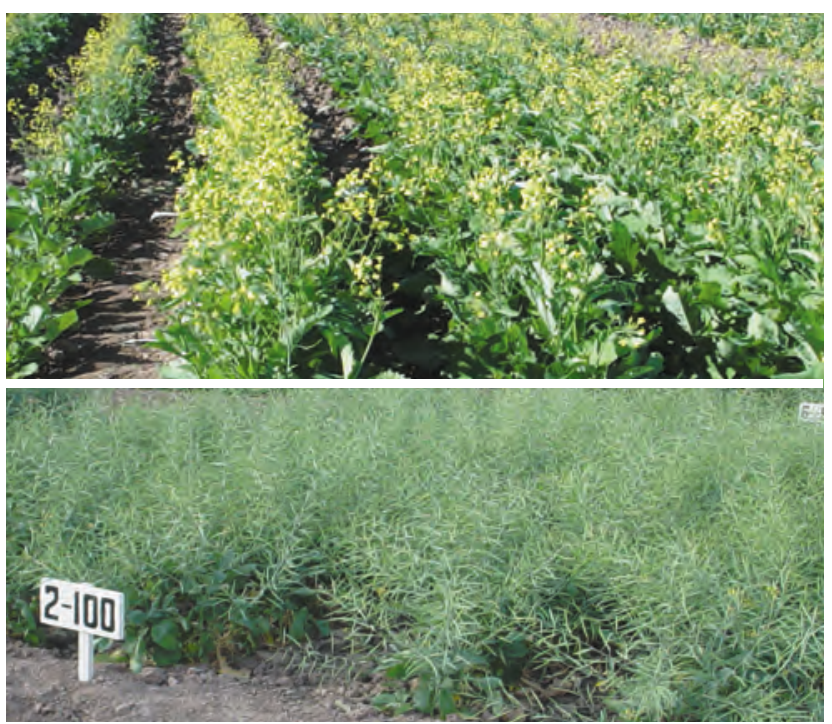

Figura 1. Cultivo de la canola híbrida 'Hyola-401'.

Cuadro 3. Tratamientos de riego ensayados en el campo para la canola.

\begin{tabular}{lcc}
\hline \multirow{2}{*}{ Tratamiento } & \multicolumn{2}{c}{ Humedad aprovechable consumida por etapa $(\%)$} \\
\cline { 2 - 3 } & Vegetativa $\left(\mathrm{HAC}_{1}\right)$ & Reproductiva $\left(\mathrm{HAC}_{2}\right)$ \\
\hline 1 & 40 & 40 \\
2 & 40 & 80 \\
3 & 60 & 60 \\
4 & 60 & 100 \\
5 & 80 & 40 \\
6 & 80 & 80 \\
7 & 100 & 60 \\
\hline
\end{tabular}

manguera provista con un medidor volumétrico calibrado para aplicar en forma precisa el volumen de agua requerido.

Se hicieron muestreos de humedad del suelo en forma continua durante todo el desarrollo de la canola. El primer muestreo fue para medir la humedad inicial antes del riego de presiembra, y los muestreos posteriores se realizaron durante el transcurso del experimento para monitorear los abatimientos de humedad del suelo en cada tratamiento. Al alcanzarse el nivel de humedad prefijado para cada tratamiento, se aplicó riego hasta reponer la capacidad de campo en el perfil de suelo de $90 \mathrm{~cm}$.

Las variables de respuesta evaluadas fueron: rendimiento de grano $\left(\mathrm{t} \mathrm{ha}^{-1}\right)$, eficiencia de uso del agua $\left(\mathrm{kg} \mathrm{m}^{-3}\right) \mathrm{y}$ agua evapotranspirada por la canola. Además se monitoreo la humedad del suelo como se apuntó antes, y se cuantificó la producción de materia seca durante el crecimiento del cultivo en cada tratamiento. Los grados día de desa- rrollo (GDD) se calcularon por medio de la metodología curva seno modificada (Allen, 1976; citado por Villa et al., 2005b); como temperaturas base y máxima a las cuales el desarrollo de la canola no es afectado, se tomó 5 y $35^{\circ} \mathrm{C}$ (Angadi et al., 2000).

Los análisis estadísticos de los datos obtenidos consistieron de análisis de varianza y comparación de medias con la prueba de Tukey $(\alpha=0.05)$. Para la obtención de los modelos estadísticos se aplicó el método de mínimos cuadrados del análisis de regresión múltiple. Todos estos análisis se hicieron con el paquete estadístico SAS (2003).

\section{RESULTADOS Y DISCUSIÓN}

\section{Condiciones de temperatura}

La temperatura media diaria observada en el ciclo de la canola de $18.9^{\circ} \mathrm{C}$ (Figura 2) que superó en $1.8^{\circ} \mathrm{C}$ a la media 
histórica de $17.1{ }^{\circ} \mathrm{C}$ para el mismo periodo. Estas fluctuaciones de clima justifican este tipo de estudios porque influyen en las necesidades hídricas de los cultivos.

La humedad aprovechable consumida en el suelo osciló de 44 a $91 \%$ durante el período de emergencia a inicio de floración, y de 43 a $99 \%$ durante el período de inicio de floración a madurez fisiológica. Estos porcentajes indican los rangos de abatimiento de humedad en el suelo que fueron operativamente posibles en el campo mediante el riego por gravedad. El consumo de agua por el cultivo fluctúo de 17.7 a $25 \mathrm{~cm}$ durante la etapa vegetativa, que es un rango de consumo de agua limitado atribuible a la escasa demanda de agua debida a las bajas temperaturas prevalecientes durante el crecimiento de la canola. El consumo de agua para la etapa reproductiva osciló de 24.1 a $40.7 \mathrm{~cm}$ de lámina de agua, etapa que ocurrió en primavera.

En los tratamientos más húmedos se propiciaron los valores más altos de disponibilidad de agua en el suelo, de 56 y $55 \%$ de la humedad aprovechable residual al momento de proporcionar el riego y llevarlo a $100 \%$ de la humedad aprovechable en el perfil de $90 \mathrm{~cm}$. El Tratamiento 1 presentó el mayor consumo de agua durante todo el ciclo con $65.7 \mathrm{~cm}$ (Cuadro 4). En contraste, el Tratamiento 7 que se sometió a la mayor restricción de humedad en la etapa vegetativa al dejar abatir la humedad aprovechable residual hasta $9 \%$, significó condiciones extremas de deficiencia de humedad en el perfil del suelo para el desarrollo de la canola. Condiciones similares de estrés hídrico se presentaron en la etapa reproductiva del Tratamiento 4 en la que el riego se aplicó hasta disminuir a $1 \%$ la humedad aprovechable residual en el suelo.

\section{Efecto de los regímenes de humedad en la canola}

Los análisis de varianza demostraron que los tratamientos impuestos provocaron diferencias significativas $(\mathrm{P} \leq$ 0.01) en ambas variables (Cuadro 4 ), y los promedios de tratamientos se muestran en el Cuadro 5 .

\section{Rendimiento de grano}

El Tratamiento 3 (61-61) mostró el rendimiento de grano más alto con $3.3 \mathrm{t} \mathrm{ha}^{-1}$, que resultó superior $(\mathrm{P} \leq 0.05)$ al resto de los tratamientos (Cuadro 5). Dicho rendimiento de canola se obtuvo al consumir 61 y $61 \%$ de la humedad aprovechable del suelo, que equivale a disponer de 39 y 39 $\%$ de la humedad aprovechable residual en las etapas vegetativa y reproductiva respectivamente, antes de aplicar el siguiente riego. La superficie de respuesta del rendimiento de grano $\left(\mathrm{t} \mathrm{ha}^{-1}\right)$ a las condiciones de humedad del suelo impuestas en los tratamientos, se ilustra gráficamente en la Figura 3.

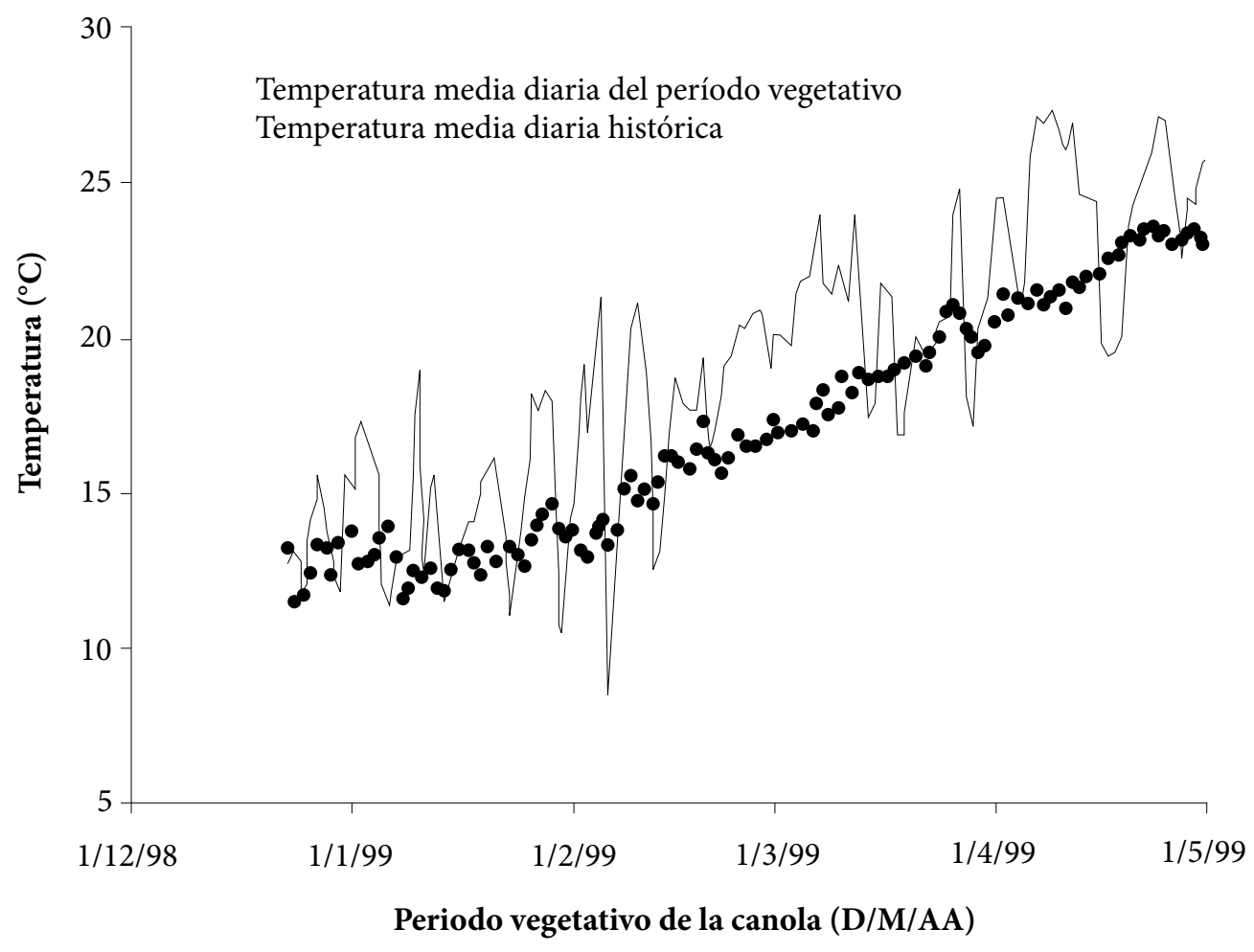

Figura 2. Climograma del periodo de crecimiento de la canola durante el estudio, comparado con la temperatura media histórica. 
Cuadro 4. Análisis de varianza y pruebas de F para rendimiento de grano y eficiencia de uso de agua de la canola.

\begin{tabular}{lccc}
\hline Fuentes de variación & GL & Rendimiento de grano $\left(\mathrm{t} \mathrm{ha}^{-1}\right)$ & Eficiencia de uso de agua $\left(\mathrm{kg} \mathrm{m}^{-3}\right)$ \\
\hline Bloques & 3 & 0.0062 & 0.0002 \\
Tratamientos & 6 & $0.8821^{\star \star}$ & $0.0214^{\star *}$ \\
Error & 18 & 0.0027 & 0.0001 \\
$\mathrm{CV}(\%)$ & & 2.0 & 2.3 \\
\hline
\end{tabular}

** Significativo al nivel de probabilidad de $0.01 . \mathrm{GL}=$ grados de libertad; CV = coeficiente de variación.

Cuadro 5. Efecto del régimen de humedad del suelo en el rendimiento promedio de grano $\left(t \mathrm{ha}^{-1}\right)$ y en la eficiencia de uso del agua $\left(\mathrm{kg} \mathrm{m}^{-3}\right)$ de canola.

\begin{tabular}{lcccccc}
\hline Tratamiento & $\mathrm{HAC}_{1}(\%)$ & $\mathrm{HAC}_{2}(\%)$ & $\mathrm{LC}_{1}(\mathrm{~cm})$ & $\mathrm{LC}_{2}(\mathrm{~cm})$ & $\mathrm{R}_{\left(\mathrm{t} \mathrm{ha}^{-1}\right)}$ & $\mathrm{EUA}\left(\mathrm{kg} \mathrm{m}^{3}\right)$ \\
\hline 1 & 44 & 45 & 25.0 & 40.7 & $2.8 \mathrm{bc}^{\dagger}$ & $0.42 \mathrm{e}$ \\
2 & 44 & 80 & 25.0 & 30.5 & $2.0 \mathrm{e}$ & $0.36 \mathrm{f}$ \\
3 & 61 & 61 & 21.4 & 35.5 & $3.3 \mathrm{a}$ & $0.58 \mathrm{a}$ \\
4 & 67 & 99 & 21.2 & 24.1 & $2.1 \mathrm{e}$ & $0.45 \mathrm{~d}$ \\
5 & 77 & 43 & 20.8 & 40.3 & $2.7 \mathrm{c}$ & $0.43 \mathrm{de}$ \\
6 & 81 & 81 & 20.3 & 31.1 & $2.9 \mathrm{~b}$ & $0.48 \mathrm{c}$ \\
7 & 91 & 65 & 17.7 & 36.7 & $2.5 \mathrm{~d}$ & $0.53 \mathrm{~b}$ \\
\hline
\end{tabular}

Medias con letras iguales en una columna no son estadísticamente diferentes (Tukey, 0.05). $\mathrm{LC}_{1}$ y LC $\mathrm{LC}_{2}$ son las láminas consumidas de agua en las etapas vegetativa y reproductiva, respectivamente; $\mathrm{R}=$ rendimiento de grano; $\mathrm{HAC}=$ humedad aprovechable del suelo consumida por la canola $(\%), \mathrm{EUA}=$ eficiencia de uso del agua de la canola.

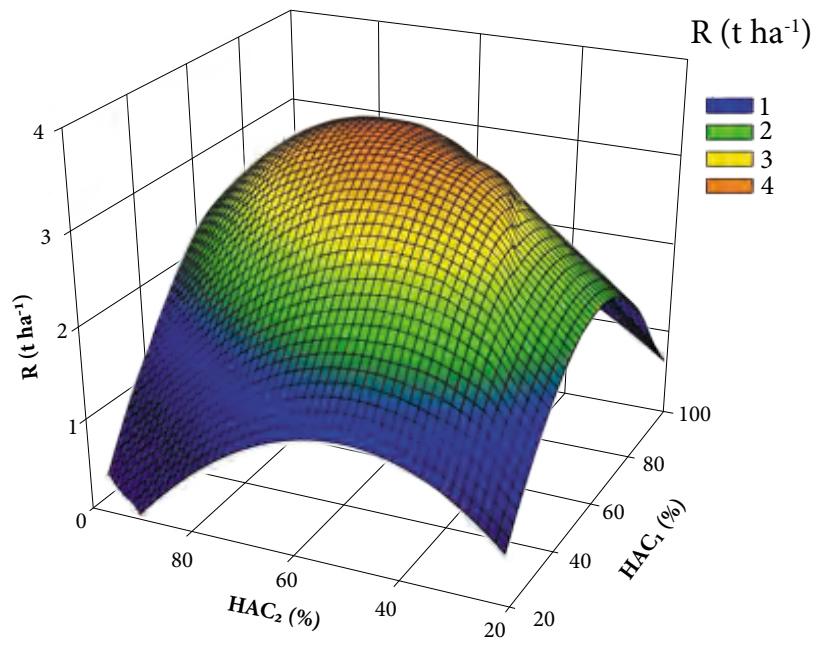

Figura 3. Superficie de respuesta del rendimiento de grano $(R)$ en función de la humedad aprovechable consumida (HAC) en dos etapas fenológicas del cultivo de la canola $\left(\mathrm{HAC}_{1}\right.$ vegetativa, $\mathrm{HAC}_{2}$ reproductiva).

Los rendimientos más bajos se registraron en los Tratamientos 2 y 4 , cuyos rendimientos fueron de 2.0 y $2.1 \mathrm{t} \mathrm{ha}^{-1}$ respectivamente, donde el cultivo estuvo sometido a déficits hídricos extremos de 80 y 99 \% de la HAC en la etapa reproductiva del cultivo. Estos rendimientos resultaron estadísticamente inferiores con respecto al resto de tratamientos $(\mathrm{P} \leq 0.05)$. Los rendimientos aquí obtenidos son similares a los reportados por Tesfamariam et al. (2010), sobre todo el más alto de $3.8 \mathrm{t} \mathrm{ha}^{-1}$.

Estos resultados permiten deducir que el rendimiento de grano de la canola tiene una respuesta no lineal ante diferentes regímenes de humedad del suelo, ya que disminuyó cuando la canola se sometió a déficits hídricos o a excesos de humedad.

\section{Eficiencia de uso de agua de la canola $\left(\mathrm{kg} \mathrm{m}^{-3}\right)$}

La mayor eficiencia productiva del agua utilizada por la canola se presentó en el Tratamiento 3, con un valor promedio de $0.585 \mathrm{~kg} \mathrm{~m}^{-3}$ (Cuadro 5), similar al valor de 0.54 reportado por Tesfamariam et al. (2010). El Tratamiento 3 corresponde a la aplicación de riego hasta que la canola haya consumido $61 \%$ de la humedad aprovechable en el suelo (HAC), tanto en la etapa vegetativa como en la reproductiva. El valor más bajo para esta variable $\left(0.36 \mathrm{~kg} \mathrm{~m}^{-3}\right) \mathrm{se}$ manifestó en el Tratamiento 2 que fue el sometido al mayor estrés hídrico en la etapa reproductiva, al reponer la humedad hasta que la planta consumió $80 \%$ de la humedad aprovechable del suelo, lo que redujo significativamente la tasa de formación de grano por cada $\mathrm{m}^{3}$ de agua utilizada. 


\section{Modelos de predicción de rendimiento de grano de canola}

\section{En función del régimen de humedad del suelo}

El modelo de simulación de la respuesta de la canola al estrés hídrico del suelo se obtuvo mediante análisis de regresión múltiple, al relacionar los datos observados del rendimiento de grano como variable dependiente y la humedad aprovechable consumida del suelo al momento del riego como variable independiente (SAS, 2003). La función de mejor ajuste (máxima $\mathrm{R}^{2}$ ) fue:

$R=-2.6954+0.1338 \times \mathrm{HAC}_{1}+0.056 \times \mathrm{HAC}_{2-} 0.0014 \times \mathrm{HAC}_{1}{ }^{2}$ $-0.0009 \times \mathrm{HAC}_{2}^{2}+0.0009 \times \mathrm{HAC}_{1} \times \mathrm{HAC}_{2}$

$\mathrm{R}^{2}=0.98, \mathrm{CV}=2.13 \%$

Donde HAC representa la humedad aprovechable del suelo consumida por la canola (\%), y $\mathrm{R}$ el rendimiento de grano de la canola $\left(\mathrm{t} \mathrm{ha}^{-1}\right)$. $\mathrm{R}^{2}$ es el coeficiente de determinación o bondad de ajuste del modelo; $\mathrm{CV}$ es el coeficiente de variación (\%); y los subíndices 1 y 2 representan las etapas vegetativa y reproductiva del cultivo, respectivamente.

Además se hizo un análisis de optimización de la función obtenida de acuerdo con la teoría de máximos y mínimos (Martínez, 1988). De este análisis se concluyó que la canola maximiza su producción de grano a $3.36 \mathrm{t} \mathrm{ha}^{-1}$ cuando se desarrolla bajo un régimen de humedad del suelo equivalente a 65 y a $61 \%$ de la humedad aprovechable consumida del suelo al momento del riego, para las etapas vegetativa y reproductiva respectivamente (Figura 3 ).

El modelo obtenido presentó un ajuste $\left(\mathrm{R}^{2}=0.98\right)$ y todos sus coeficientes de regresión fueron significativos $(\mathrm{P} \leq$ 0.01). Inzunza et al. (2010) reportaron resultados similares de aplicar el riego a $65 \%$ de la HAC durante todo el ciclo de la canola.

\section{En función de la evapotranspiración o lámina de agua consumida}

Al relacionar la producción de grano de la canola con la lámina de agua consumida, se encontró que entre ambas variables existió una relación de tipo cuadrática con rendimientos crecientes y decrecientes, como se ilustra en la Figura 4.

La función de mejor ajuste obtenida por análisis de regresión entre ambas variables (SAS, 2003) resultó como sigue:
$R=-9.3723+0.8606 \times L C_{1}+0.1926 \times L C_{2}-0.0397 \times L C_{1}{ }^{2}$
$0.0101 \times L C_{2}{ }^{2}+0.0239 \times L C_{1} \times L C_{2}$

$\mathrm{R}^{2}=0.92, \mathrm{CV}=5.2 \%$

Donde: $\mathrm{R}=$ rendimiento de grano de canola; $\mathrm{LC}_{1}=$ lámina de agua consumida en la etapa vegetativa; $\mathrm{LC}_{2}=$ lámina de agua consumida en la etapa reproductiva; $\mathrm{R}^{2}=$ coeficiente de determinación; $\mathrm{CV}=$ coeficiente de variación.

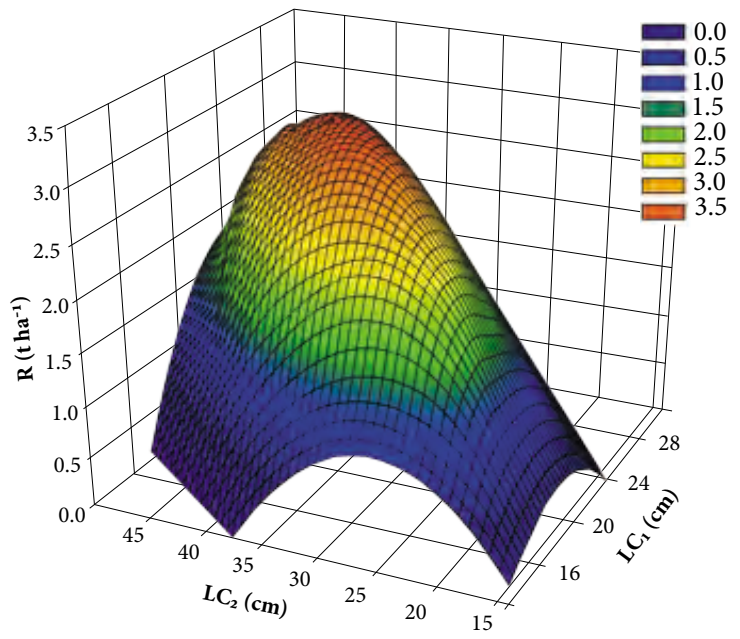

Figura 4. Superficie de respuesta del rendimiento de canola $(R)$ en función del agua consumida durante la etapa vegetativa $\left(L C_{1}\right)$ y la reproductiva $\left(L C_{2}\right)$.

Del respectivo análisis de optimización de la función efectuado de acuerdo con la teoría de máximos y mínimos (Steel et al., 1997), se concluyó que la canola maximiza su producción de grano a $3.15 \mathrm{t} \mathrm{ha}^{-1}$ cuando consume láminas de agua de 21.3 y $34.8 \mathrm{~cm}$ durante su desarrollo vegetativo y reproductivo, respectivamente. En el Estado de Colorado, EE. UU., Nielsen (1997) reportó una lámina de $52.1 \mathrm{~cm}$ para canola, y en Sudáfrica Tesfamariam (2010) reportó un consumo de $55 \mathrm{~cm}$ en todo el ciclo vegetativo de la misma especie, en ambos casos con rendimientos muy similares a los observados en el presente estudio.

Cabe notar, tal como se esperaba, que los rendimientos máximos estimados con las Ecs. 1 y 2 fueron similares (3.36 vs. $3.15 \mathrm{t} \mathrm{ha}^{-1}$ ) debido a que las variables independientes HAC y LC están altamente correlacionadas entre sí.

\section{Producción de biomasa}

La mayor producción de materia seca se registró en el Tratamiento 1 (40-40) cuando el cultivo alcanzó 685 grados día de desarrollo (GDD) a los $94 \mathrm{~d}$ después de emergencia (Figura 5). En este mismo periodo todos los tratamientos 


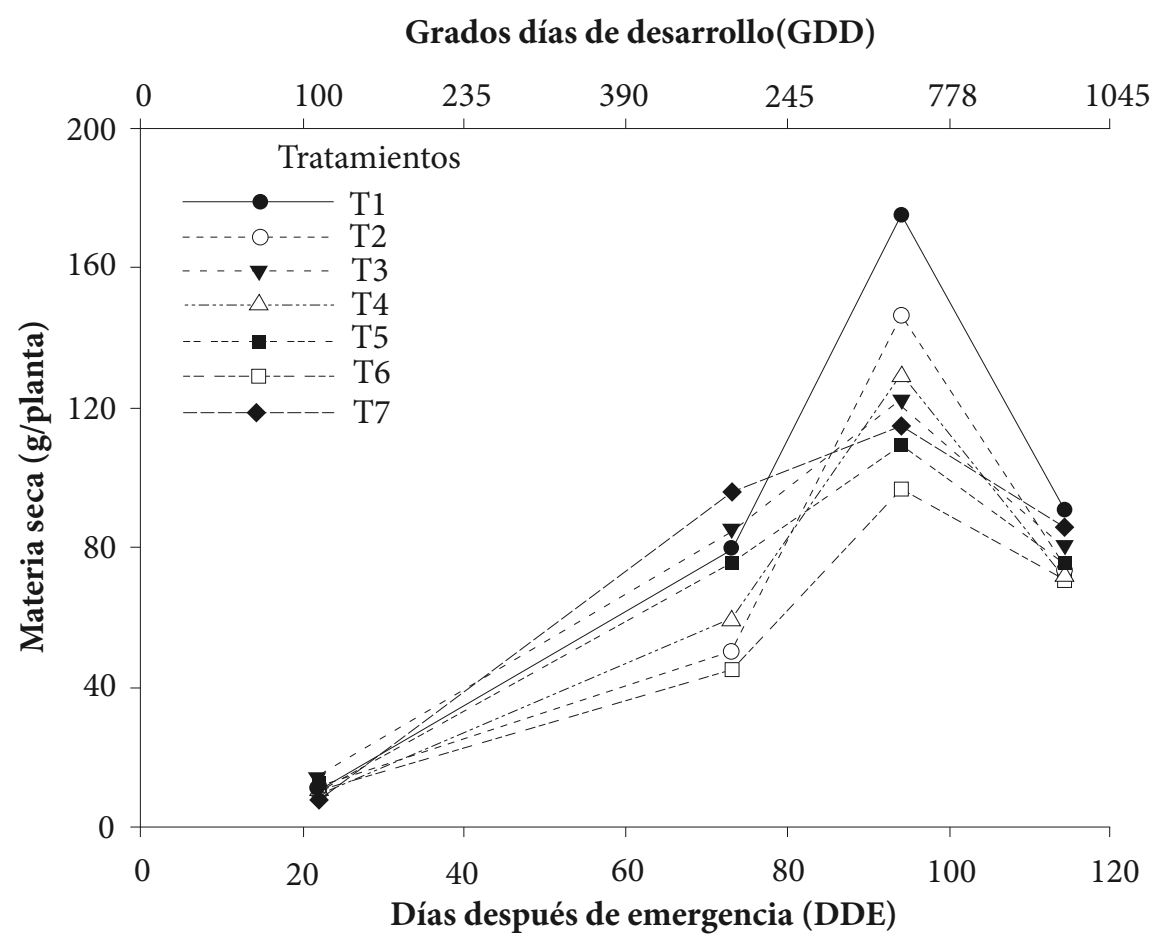

Figura 5. Cinética de producción de materia seca de la canola en siete tratamientos de estrés hídrico.

también alcanzaron la mayor producción de materia seca y el mayor crecimiento.

El Tratamiento 3 (60-60) que tuvo el mayor rendimiento de grano, acumuló menos biomasa que la registrada en los tratamientos que dispusieron de más humedad en la etapa vegetativa (40-40 y 40-80), que es cuando se define y se genera más de $95 \%$ del crecimiento y de la cobertura vegetal. Se infiere entonces que la mayor producción de grano de la canola no coincide con su mayor cobertura, como lo han reportado otros investigadores para cultivos oleaginosos y cereales. Esto se atribuye a que en canola los fotoasimilados se canalizan más a la producción de biomasa que al grano, como se muestra en la Figura 6. Resultados similares fueron reportados por Villa-Castorena et al. (2007), quienes aplicaron un modelo logístico con un ajuste de $\mathrm{R}^{2}=0.90$.

\section{CONCLUSIONES}

Los modelos de simulación generados tuvieron un ajuste mayor de $90 \%$ y un coeficiente de variación menor de 8 $\%$. Con el modelo optimizado que relaciona el rendimiento de grano y la humedad aprovechable del suelo, la canola logró su mayor producción $\left(3.36 \mathrm{t} \mathrm{ha}^{-1}\right)$ al consumir $65 \mathrm{y}$ $61 \%$ de la humedad aprovechable del suelo al momento del riego, durante las etapas vegetativa y reproductiva, respectivamente. Con la función optimizada que predice la producción de grano en función de la cantidad de agua consumida por el cultivo, la canola alcanzó su máximo rendimiento de grano (3.15 $\mathrm{t} \mathrm{ha}^{-1}$ ) al consumir 21.3 y $34.8 \mathrm{~cm}$ de agua durante las etapas vegetativa y reproductiva, respectivamente. El valor más alto de la eficiencia de uso del agua de $0.585 \mathrm{~kg}$ $\mathrm{m}^{-3}$ por la canola ocurrió en el tratamiento con un consumo de $61 \%$ de la humedad aprovechable, tanto en la etapa vegetativa como en la reproductiva, similar a los valores óptimos observados.

La producción de biomasa también resultó afectada por las condiciones de estrés hídrico aplicado en los diferentes tratamientos. La respuesta de esta variable a la disponibilidad de agua fue lineal y diferente a la del rendimiento de grano, debido a que el cultivo canalizó preferentemente los fotoasimilados a la producción de biomasa que al grano.

\section{AGRADECIMIENTOS}

Al Consejo Nacional de Ciencia y Tecnología (CONACYT), por el apoyo económico otorgado mediante el proyecto núm. 90744. 


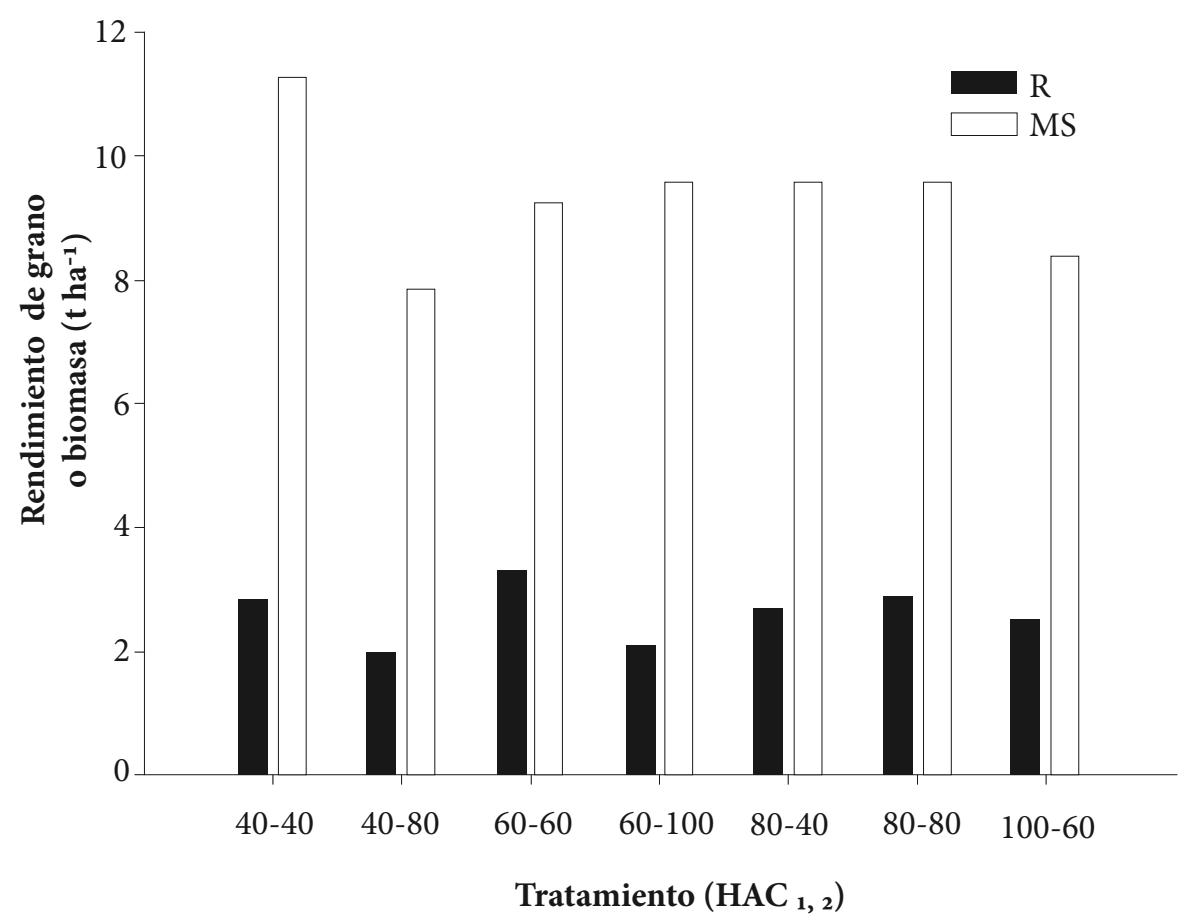

Figura 6. Comportamiento del rendimiento de grano $(\mathrm{R})$ y de la producción de biomasa (MS) de canola, en siete tratamientos de riego. $\mathrm{HAC}_{1,2}$ = humedad aprovechable consumida en el suelo en la primera (vegetativa) y segunda etapa (reproductiva).

\section{BIBLIOGRAFÍA}

Al-Ghobari H. M. (2000) Estimation of reference evapotranspiration for southern region of Saudi Arabia. Irrigation Science 19:81-86.

Allen J. C. (1976) A modified sine wave method for calculating degree days. Environmental Entomolowgy 5:388-396.

Angadi S. V., H. W. Cutforth, B. G. McConkey, M. H. Entz, K. Volkmar and S. Brandt (2000) Response of three Brassica species to high temperature injury during reproductive growth. Canadian Journal of Plant Science 80:693-701.

Booth E. J. and F. D. Gunstone (2004) Rapeseeds and rapeseed oil: Agronomy, production, and trade. In: Rapeseed and Canola Oil Production, Processing, Properties and Uses. F D Gunstone (ed). Blackwell Publishers. Oxford, UK. pp:1-16.

Blum A. (2005) Drought resistance, water use efficiency and yield potential. Are they compatible, dissonant or mutually exclusive? Australian Journal of Agricultural Research 56:1159-1168.

Castellanos R. J., B. S. Uvalle and S. A. Aguilar (2000) Manual de Interpretación de Análisis de Suelos y Aguas. 2a ed. Colección INCAPA. San Miguel de Allende, Gto. 226 p.

Chongo G. and P. B. McVetty (2000) Relationship of physiological characters to yield parameters in oilseed rape (B. napus). Canadian Journal of Plant Science. 81:1-6.

CONAGUA, Comision Nacional del Agua (2011) Estadísticas Agrícolas de los Distritos de Riego. Año Agrícola 2009-2010. México, D.F. $317 \mathrm{p}$.

Faraji A., N. Latifi, A. Soltani and A. H. Shirani (2009) Seed yield and water use efficiency of canola (Brassica napus L.) as affected by high temperature stress and supplemental irrigation. Agricultural Water Management 96:132-140.

Gan Y., S. V. Angadi, H. Cutforth, D. Potts, V. V. Angadi and C. L. McDonald (2004) Canola and mustard response to short periods of temperature and water stress at different developmental stages. Canadian Journal of Plant Science 84:697-704.
García E. (1988) Modificaciones al Sistema de Clasificación Climática de Köppen. Offset Larios, México. 217 p.

Inzunza-Ibarra M. A., E. C. Catalán-Valencia, M. M. Villa-Catorena, I. Sánchez-Cohen and A. Román- López (2010) Respuesta de la canola al déficit hídrico del suelo. Revista Fitotecnia Mexicana 33:53-59.

Johnston A. M., D. L. Tanaka, P. R. Miller, S. A. Brandt, D. C. Nielsen, G. P. Lafond and N. R. Riveland (2002) Oilseed crops for semiarid cropping systems in the northern Great Plains. Agronomy Journal 94:231-240.

Kassam A. H., D. Molden, E. Fereres and J. Doorenbos (2007) Water productivity: science and practice-introduction. Irrigation Science 25:185-188.

Martínez G. A. (1988) Diseños Experimentales. Métodos y Elementos de Teoría. Ed. Trillas. México, D.F. 752 p.

Monteith J. L. (1977) Climate and the efficiency of crop production in Britain. Phil. Trans. R. Soc. London. Ser. B 281:277-294.

Nielsen D. C. (1997) Water use and yield of canola under dryland conditions in the Central Great Plains. Journal of Production Agriculture 10:307-313.

Plenecassage A., E. Romero-Fierro y C. López-Borrego (1997) Manual de Laboratorio. Análisis de Suelos y Aguas. INIFAP-ORSTROM. Gómez Palacio, Durango, México. 173 p.

Si P. and G. H. Walton (2004) Determinants of oil concentration and seed yield in canola and Indian mustard in the lower rainfall areas of Western Australia. Australian Journal of Agricultural Research 55:367-377.

SAS, Statistical Analysis System (2003) (SAS/STAT) 2003.9.1 by SAS Institute Inc. Cary, USA.

Steel R G., J. H. Torrie and D. A. Dickey (1997) Principles and Procedures of Statistics. A Biometrical Approach. 3rd ed. The McGrawHill Companies, Inc. 666 p.

Tesfamariam H. E., J. G. Annandale and J. M. Steyn (2010) Water s stress effects on winter canola growth and yield. Agronomy Journal 102:658-666. 
Villa-Castorena M. M., E. A. Catalán-Valencia y M. A. Inzunza-Ibarra (2005a) Análisis de la información climática para usos agrícolas. Agrofaz 5:717-724.

Villa-Castorena M. M., E. A. Catalán-Valencia y M. A. Inzunza-Ibarra (2005b) Evaluación de la metodología curva seno modificada para estimar grados-día en tres localidades del norte de México. Agrofaz 5:851-856.
Villa-Castorena M. M., E. A. Catalán-Valencia, A. Román-López, M. A. Inzunza-Ibarra, S. F. Mendoza-Moreno, J. J. Duarte, A. D. Báez y B. Gómez-Lucatero (2007) Parámetros fisiotécnicos y requerimientos agroclimáticos de la canola (Brassica napus L.). Agrofaz 9:13-19.

Villasmil P. J., E. Martínez y G. Segura (1974) El diseño San Cristóbal y su uso en ensayos de fertilización en caña de azúcar. + (LUZ) 3:7-25. 